\title{
The interrelation between aerobic exercise, mental well- being, stress response and epigenetics
}

\author{
Feller $\mathrm{L}^{1}$, Nemutandani MS ${ }^{1}$, Feller $\mathrm{G}^{2}$, Jose $\mathrm{RJ}^{3}$, Lemmer $\mathrm{J}^{4}$ and Khammissa RAG ${ }^{5 *}$ \\ ${ }^{1}$ School of Oral Health Sciences. University of the Witwatersrand, South Africa \\ ${ }^{2}$ Department of Radiation Oncology, University of Witwatersrand, South Africa \\ ${ }^{3}$ Royal Brompton Hospital, United Kingdom \\ ${ }^{4}$ Department of Periodontology and Oral Medicine, University of the Witwatersrand, South Africa \\ ${ }^{5}$ Department of Periodontics and Oral Medicine, School of Oral Health Sciences, University of Pretoria, South Africa
}

\begin{abstract}
Mental well-being is a subjective, adaptive state of mind arising from complex dynamic interactions between cognitive, emotional and psychosocial factors. Positive mental well-being may promote good physical health, occupational functioning and academic achievement. In the moderate-to-vigorous intensity of aerobic range, regular cardiorespiratory exercise of sufficient duration and frequency has the capacity to boost resources of mental energy, to improve sleep, mental alertness and social interactions, to buffer maladaptive upregulated emotional responses to psychosocial stressors, to decrease anxiety, to moderate depressive symptoms and to support or even improve cognitive functioning.
\end{abstract}

Epigenetic modification is a biological mechanism by which environmental factors influence cellular phenotype and function. Psychosocial stressors, and regular performance of aerobic exercise have opposite effects on the central nervous system, mediated at least in part through epigenetic modifications that reprogram the expression of relevant genes.

Generally, physically active persons are more resilient to stress and experience fewer depressive symptoms than do physically inactive persons; and, indeed, regular moderate to strenuous aerobic activity has a mood-enhancing effect and has been proven to mitigate negative affectivity stress responses.

The purpose of this narrative review is to discuss the mechanisms that appear to play a role in aerobic exercise-induced improvement in mental well-being.

\section{Introduction}

Physical fitness refers to the capacity to perform efficiently and effectively all routine daily activities, whether of work or leisure, without undue fatigue; and to be able adaptively to cope with unexpected or urgent physical demands. It is generally accepted that sleep, rest, healthy nutrition and regular moderate-intensity aerobic physical activity of sufficient duration and frequency, all contribute to functional physical fitness, and to promoting mental fitness and wellbeing. To be of any physiological value to the average healthy person, aerobic physical activity should increase both heart and respiratory rates sufficiently to accelerate the blood circulation, with consequent increased tissue oxygenation and cardiorespiratory fitness [1].

In general, aerobic exercise refers to the physical activity of large muscle-groups, utilizing oxygen in the process of cellular respiration whereby carbohydrates, fat and proteins, ultimately by way of adenosine triphosphate (ATP) produce the immediate energy necessary to sustain continuous activity that lasts more than one minute. The efficacy of this oxidative phosphorylation metabolic pathway is dependent on the capacity of the cardiorespiratory system to supply the necessary oxygenated haemoglobin, and of the skeletal muscles to deploy this via their microvasculature [2,3]. Although oxidative phosphorylation is critically important, it is not the exclusive source of energy for aerobic exercise; to a minor extent the anaerobic glycolysis and high energy phosphate metabolic pathways also contribute to the energy required for the performance of aerobic exercise [3].
Aerobic intensity is a function of the cardiorespiratory effort associated with performance of everyday activities, or of deliberate, purposeful exercise, and can be directly related to the heart rate and oxygen consumption during the physical effort [4]. A measure of the intensity of aerobic exercise can be determined by the heart rate during the activity.

A heart rate of $50 \%$ to $70 \%$ above the maximum person specific normal rate is regarded as indicative of moderately intense exercise, while a rate that is $70 \%$ to $85 \%$ above the normal maximum denotes vigorously intense exercise. This varies with gender, age, general health and level of fitness [5]. In order substantially to benefit physical and mental health, an adult should undertake at least 150 to 300 minutes of moderately intense, or 75 to 150 minutes of vigorously intense aerobic exercise per week, or some combination of both. Regular anaerobic exercise also gives rise to additional health benefits (Piercy $\&$ Troiano, 2018). In general, compared to non-exercisers, those who exercise regularly and are aerobically fit show reduced physical and psychological reactivity to psychosocial stressors [6-8].

${ }^{\star}$ Correspondence to: Razia Khammissa, Head, Department of Periodontics and Oral Medicine, School of Dentistry, Faculty of Health Sciences, University of the Pretoria, South Africa, Tel: +27-827863228; E-mail: razia.khammissa@up.ac.za

Key words: aerobic fitness, psychosocial stressors, stress responses, cognitive functioning, epigenetic modifications, endocannabinoid system, mental well-being Received: July 05, 2021; Accepted: August 20, 2021; Published: August 27, 2021 
Aerobic exercise of moderate to vigorous intensity (MVAE), and psychosocial stressors, activate the autonomic nervous system and the neuroendocrine system similarly, eliciting signs and symptoms of physiological arousal [8] including increased blood pressure and respiratory rate and perspiration. Therefore, regular MVAE may habituate body and mind to the commonality of the arousal effects generated by both aerobic exercise and psychosocial stressors, so that the subject should be able to recognize and appraise the effects of psychosocial stressors as being challenges rather than potential threats, thus buffering the maladaptive exaggerated responses to psychosocial stressors, and improving mental well-being [9-11]. In contrast to MVAE, light physical activities (house-work, shopping, strolling) which do not raise the heart and respiratory rate significantly, do not seem to bring about any appreciable health benefits; and sedentary behavior characterized by little physical activity and much sitting during wake time is neither beneficial to mental or physical health [1].

Nonetheless, recreational or light leisure-time physical activities such as walking, cycling, swimming, yoga, or tai chi, which require only moderate cardio-respiratory effort, may enhance mental wellbeing because they bring about feelings of enjoyment, competence, a fresh outlook on things, and improved body image and selfesteem. Moreover, they allow time away from fatiguing exposure to environmental and psychosocial stressors and negative preoccupying thoughts and provide an opportunity to socialize with friends and to spend quality time with family members $[6,12-14]$.

Through different mechanisms, aerobic exercise of adequate intensity, duration and frequency has the capacity to induce central neurological adaptations that may moderate responses to psychosocial stressors, reduce symptoms of anxiety and depression, and may improve cognitive functioning (working memory, cognitive flexibility, information processing and inhibitory control), and mental wellbeing [15-18]. Furthermore, MVAE appears to have the capacity to attenuate the development of age-related neurodegenerative processes and the consequent decline in cognitive functions; and to retard the progression of neurocognitive diseases such as Parkinson disease and Alzheimer disease $[18,19]$.

By epigenetic modifications, MVAE activates a number of intracellular signalling pathways which stimulate transcription factors, and subsequently initiate transcriptional regulation of genes encoding biochemical agents that mediate a variety of biological functions $[18,20]$. Psychosocial stress induced aberrations in epigenetic patterns of DNAmethylation and histone modifications, with consequent dysfunctional regulation of expression of genes encoding central neurotransmitters, neurotrophic proteins and neuroendocrine peptides and hormones, collectively contribute to stress-induced cognitive deficits and to poor mental well-being. With its capacity to ameliorate the epigenetic imbalances induced by psychosocial stressors, MVAE reverses or counteracts these deficits, and improves cognition and mental wellbeing $[18,21]$.

Thus, epigenetic modifications constitute the biological means whereby environmental factors such as occupational or social stress, physical exercise or lack thereof, nutrition, pollutants and others have the capacity to influence gene expression and cellular functioning, and to contribute to one's inherited mental predisposition [22]. However, little is known about the actual genetic, epigenetic and molecular mechanisms involved in bringing about the mental health benefits mediated by MVAE; or about the factors that account for the different effects of the same MVAE program upon persons of comparable age, physical and mental health and environmental circumstances.
The purpose of this narrative review is to discuss the various mechanisms whereby environmental stress and aerobic exercise may oppositely affect physical and mental well-being; and to draw attention to the importance of including aerobic exercise among the means used to promote positive physical and mental well-being. An attempt has been made critically to analyze relevant databases and authoritative texts and to integrate the information to better understand these mechanisms.

\section{The concept of mental well-being}

Mental well-being can be conceptualized as a dynamically judgmental view of one's quality of life, and general satisfaction with the fulfillment of one's unfolding expectations of life. The complex interplay between gender, age genetic, epigenetic and environmental factors determine overall subjective spatial, dimensional and temporal perceptions of mental well-being. Adverse life events, poor stress-coping capacity, negative thoughts and emotions, rumination, unresolved emotional stress, anxiety, depression, chronic pain or chronic illness and lack of social support are some common factors associated with poor or negative mental well-being. On the other hand, good physical health, functional neurocognitive mechanisms (resilience, coping, volition, self-control, etc.), competence, relatedness and social support, and a sense of self-realization and existential meaningfulness are some important factors associated with positive mental well-being. In order to achieve a consistently positive mental health status (Table 1), one needs both to play down the negative, and to nurture the positive factors associated with mental well-being $[12,23,24]$.

Thus, the subjective broad conception of mental well-being is determined by positive experiences, interesting and meaningful activities, pleasant and upbearing emotions, satisfied psychological and biological needs, and by low levels of negative emotions and thoughts [25]. Since judgments about one's own life events are necessarily subjective, the conception of mental well-being differs from person to person, and usually fluctuates according to each person's feelings, emotions, thoughts, experiences and mood at the time [24].

The distinctive attribute of humility is associated with positive mental well-being. Humility refers to the qualities of being aware of and acceptant of one's own limitations, biases and strengths, and of the beliefs, needs and practices of other persons. Humility also refers to willingness and the mental ability to moderate one's beliefs, values and attitudes in the light of evolving new evidence or circumstances, and to care for and respect the priorities and behaviors of others. Humble persons, therefore, may find it easier to manage maladaptive responses to psychosocial stressors and thus to lessen or moderate the impact of stress on mental well-being $[26,27]$.

It appears that gender, age and level of education do not play an important role in influencing mental well-being [24]; but good

Table 1. Some factors associated with positive mental well-being [24]

Living in democratically governed countries, in which human rights and personal security are respected and promted, and which have effective, functional health systems Personal good health, healthy lifestyle

Enjoyable and meaningful work, satisfaction with family life, financial security

Supportive social relationships

Satisfied psychological needs of self-determination (autonomy, competence, relatedness)

Natural positive thinking, emotional maturity and spirituality as intrinsic motivators of adaptive behaviour

Humility

Personality trait: extroversion

Other genetic hereditary characteristics (temperament) 
physical health, effective occupational functioning, and educational achievements are associated with positive mental well-being $[23,25]$. Conversely, experiencing psychological stress owing to unresolved emotional conflicts constitutes a major threat to mental well-being. Persistent exposure to psychosocial stressors may so dysregulate stress responses that over time they may become hyperactive, with detrimental physiological and behavioral changes weakening psychological resilience, with the potential negatively to affect mental and physical well-being, and longevity.

Thus, depending on the nature of the psychosocial stressor, and on the circumstances under which exposure to the stressor occurs, functionally adaptive stress responses may gradually evolve into selfdefeating maladaptive behavioral responses [28].

From all this, it is clear that mental well-being can be profoundly influenced by the interrelations between central neural functioning, cognition, motivation, emotionality and impulsiveness or inhibited behavior [29].

\section{Psychological mechanisms contributing to exercise-in- duced positive mental well-being}

In order to experience positive mental well-being, the psychological needs of autonomy, competence and relatedness have to be satisfied, and work-related activities must be perceived as being meaningful $[30,31]$. The impetus to goal-directed activities which require problemsolving skills, conceptual understanding, creativity, or cognitive flexibility, is autonomously intrinsically motivated by energizers such as interest, authenticity and meaningfulness that are directly derived from the activity itself. Intellectual challenge, making a contribution to society, altruism and professional or work-related satisfaction, communications with clients/patients/customers and/or colleagues are some other autonomous intrinsic motivators contributing to positive mental well-being [32,33].

Competence refers to the efficacy and mastery of goal-directed activities, and to the ability to self-improve and to develop skills and capabilities; and relatedness refers to self-selected interpersonal trusting and caring relationships with others. Both competence and relatedness promote positive mental well-being [33-37].

Regular aerobic exercise provides an opportunity to satisfy the psychological needs of autonomy, competence and relatedness, through purposeful, constructive, meaningful and beneficial activity, which, especially if done as a group-activity may also reduce social isolation and promote feelings of empowerment [38]. Compliance with any selfimposed or group-imposed regimen of MVAE may induce and sustain feelings of self-efficacy, which may improve one's subjective perception of being able to accomplish a task at hand. With enhanced self-efficacy there is an increase in self-confidence and self-control, and in perceived ability to engage successfully with other goal-directed tasks [10,39]. All these exercise-induced psychosocial benefits may have the outcome of improved mental well-being $[38,39]$.

The psychological ability to get satisfaction and enjoyment from, and regularly to engage in demanding vigorous aerobic exercise is determined in part by genetic factors. For example, some polymorphic variants of the brain-derived neurotrophic factor (BDNF) gene, and of genes encoding for certain adrenergic receptors, can influence the feeling of satisfaction, enjoyment and intrinsic motivation that come from engaging in strenuous aerobic exercise [40].

Endurance aerobic exercise has the capacity to induce the positive psychological experience of 'flow', which is characterized by being effortlessly, almost unconsciously engaged and absorbed in the activity at hand, while feeling effective, inspired and fulfilled [41]. This exercise-induced, transient, but gratifying autotelic or self-rewarding experience allows for the conscious and subconscious integration of information, reflexes and drives. Thus, as a result, the task or activity at hand is performed with minimal deliberation and metacognitive processes, and with heightened quality of cognitive and motoric performance [39,41,42].

Although the biological mechanisms that drive the flow experience are not well understood, it appears that endocannabinoidergic central neural circuits play an important role in the generation of the immediate feelings of mental well-being and euphoria associated with the flow experience following endurance exercise $[42,43]$.

\section{Some functions of the neuroendocrine and autonomic neural systems in relation to stress responses}

Moderate to vigorous aerobic exercise (MVAE) is a physical stressor that above a certain person-specific threshold of intensity disturbs the internal stability of biological systems, so that in order to maintain homeostasis, both physiological and behavioral adaptations are necessary [44]. As is the case with psychosocial stressors, MVAE elicits complex but coordinated interactions between autonomic neural, neuroendocrine and immune stress-induced processes regulated by the limbic system and the prefrontal cortex $[7,8,28,45,46]$. The outcome of these complex interactions is a consolidated stress response which is determined by the nature of the stressor (type, frequency, intensity, duration); and is modified by genetic, epigenetic and environmental factors, by one's mental and physical fitness, by cognitive appraisal of the stressor, by stress-coping capacity and by executive functioning $[8,47,48]$.

Responses to acute and chronic stressors are driven by the catecholamines adrenaline and noradrenalin which are released by the sympathetic division of the autonomic nervous system; and by neuropeptides/hormones of the hypothalamus-pituitary-adrenal (HPA) axis, including hypothalamic corticotrophin releasing hormone (CRH), arginine vasopressin (AVP), the pituitary proopiomelanocortin (POMC)-derived peptides, adrenocorticotrophic hormone (ACTH), $\alpha$-melanocyte stimulating hormone, and $\beta$-endorphin, and the adrenal cortex-derived glucocorticoids (cortisol) $[45,47,49,50]$.

The sympathetic division of the autonomic nervous system comprises the sympatho-adrenomedullary axis which mediates an increase in circulating levels of adrenaline, primarily from the adrenal medulla, and the sympatho-neural axis which increases noradrenalin out-flow, primarily from sympathetic nerves; both axes trigger rapid physiological adaptations in response to stress $[28,45,46,51]$. While the sympatho-neural axis primarily regulates blood pressure, and blood flow to the brain, the sympatho-adrenomedullary axis primarily mediates responses to serious threats to the body such as hemorrhagic hypotension, hypoglycemia, shock, and the stress of extreme-endurance physical exercise [46]. The physiological effects of activation of the parasympathetic division of the autonomic nervous system are usually opposite to those of the sympathetic nervous system; parasympathetic activation quickly downregulates any excessive sympathetic activity and moderates the sympathetic response to stress [45].

An acute stress response occurs when the amygdala of the limbic system interprets any internal or external signal as a threat to homeostasis [45]. Immediately and automatically, without conscious awareness, a distress signal is then sent from the amygdala to the dorsomedial 
hypothalamus (DMH) which in turn activates the sympathetic nervous system, with the secretion of high-levels of adrenaline from the adrenal medulla. After the surge of adrenaline subsides, the paraventricular nucleus (PVN) of the hypothalamus activates the HPA axis, resulting in secretion of cortisol, the primary stress hormone, from the adrenal cortex, into the blood stream [49-52]. Neuronal inputs from DMH thus play an important role in PVN activation and hence in the output of the HPA axis [52].

By activating corticosteroid receptors in the hypothalamus, hippocampus, amygdala and medial prefrontal cortex, the increased level of cortisol suppresses the secretion of the hypothalamic CRH and the pituitary ACTH, thus completing the negative feedback loop of the HPA axis, resulting in inhibition of further release of cortisol from the adrenal cortex [45,53-57].

This initial stage of a stress response brings about an increase in the heart and respiratory rates, in blood pressure, and in mobilization of energy; and there is increased conscious awareness, alertness, vigilance and information processing, which enable alarm-related behavior such as the 'fight-or-flight' response [7].

The major central effectors of the stress response are noradrenalin, $\mathrm{CRH}$ and POMC-derived neuropeptides, and the major peripheral effectors are cortisol and adrenaline [44,48]. However, cortisol also plays an important role centrally, inducing complex changes in central glutaminergic excitatory signaling in the prefrontal cortex, including modulation of presynaptic release of glutamate, of the functional activity of postsynaptic glutamate receptors, and of epigenetic regulation of expression of certain genes operating stress-mediated signaling pathways; and in facilitating synaptic activity of neural circuits in the amygdala, hippocampus and the prefrontal cortex, thus enabling the generation of perceptible stress responses: alarm, reaction and exhaustion [58].

If the activity of the HPA axis and of the autonomic neural activity continue for too long, the adverse effects of hyperactive maladaptive stress responses will follow [7]. These include anxiety, depression, occupational burnout and cognitive impairment, associated with increased levels of cortisol $[7,28]$; and cardiovascular dysfunction and states of hyperarousal, restlessness, agitation and poor coordination, associated with the persistently increased levels of adrenaline and noradrenalin [50].

There are some differences between stress responses to regular, moderate to vigorous aerobic exercise (MVAE), and to unabating psychosocial stressors. In the former, the increased levels of cortisol ebb away shortly after the end of the exercise; but in the latter, the cortisol levels may remain abnormally high for a long time, with the potential to cause detrimental mental effects, associated with the different patterns of central neurochemical release induced by MVAE and by psychosocial stressors $[45,50,54]$. Furthermore, while chronic psychosocial stress may impair cognitive functioning, memory and performance of goal-directed tasks (Feller et al., 2020, LeBlanc, 2009), long-term regular MVAE improves these mental capacities [10,54].

There is a reciprocal interaction between the neuroendocrine system and the immune system, with cortisol regulating immunoinflammatory responses, and the immune system via cytokines and other biological mediators secreted by immunocytes, modulating the activity of the neuroendocrine system. MVAE can influence the functional activity of both systems $[11,44,47]$.

Persistent exposure to chronic psychosocial stressors may dysregulate the activity of the immune system and increase levels of cytokines and other biological mediators that in turn can modulate the function of the neurotransmitter and the neuroendocrine systems, adversely affecting central synaptic plasticity and neural circuits. All these influence the regulation of mental activities and well-being [22].

Animal studies show that repeated exposure to the same aversive stimulus (homotypic stress) appears to be associated with a gradual reduction in the reactivity of the HPA axis and consequent in the glucocorticoid secretion in response to the same stressor. This 'stress habituation' is an adaptive mechanism that facilitates the maintenance of homeostasis by reducing glucocorticoid burden, but the neural circuits operating this mechanism of habituation are not well understood [28]. This is the opposite of what occurs with intermittent exposure to different (heterotypic) stressors, which evokes more severe changes because there is no habituation to the stressor.

As MVAE is an acute stressor which activates the autonomic nervous system and the HPA axis, it is probable that regular MVAE will optimize the functional activity of the autonomic neural, neuroendocrine, and immunoinflammatory stress systems with consequent moderation of psychological responses and improved adaptivity, tolerance and resilience to intermittent psychosocial and physical stressors [7]. The activation of the neuroendocrine and the autonomic nervous systems by MVAE also plays an important role in the development of physical and aerobic fitness [44].

Chronic exposure to psychosocial stressors may induce central neurochemical and neurostructural changes in brain regions involved in the mediation of autonomic neural and neuroendocrine stress responses, which may dysregulate the functional activity of the HPA, sympatho-adrenomedullary and sympatho-neural axes, with the generation of maladaptive stress response. Stressor-specific maladaptive responses are determined by multiple factors, including the nature of the stressor (acute, chronic, psychosocial, physical, intensity), the environmental context, the emotional burden of previous stress experiences/responses, and one's mental coping capabilities $[28,45,51]$. As the central neural circuits that operate stress responses and reward overlap to some degree, engagement with natural rewardmediated behavior such as MVAE may moderate the deleterious effects of chronic exposure to psychosocial stress [45].

\section{Neurobiology of aerobic exercise in relation to mental well-being}

Physical exercise above a certain threshold of cardiorespiratory effort increases cerebral blood flow and oxygen supply and has the capacity to induce neural plasticity with beneficial and demonstrable changes in structure and function of certain brain regions [5961]. MVAE-mediated central cellular alterations include increased angiogenesis, synaptogenesis and neurogenesis; molecular alterations include modulation of some neurotrophic agents, neurotransmitters and receptors; and structural changes include increased gray matter in the hippocampus and in the frontal cortex $[7,49,62]$.

All these exercise-mediated changes may not only slow down neurodegenerative processes, protect against age-related decrease of hippocampal volume and consequent cognitive decline, but also improve cognitive performance (executive control, memory, attention and learning), sleep patterns, mood, perceived self-esteem, energy levels and mental alertness; and together with inducing reduction in tiredness, anxiety and depression, exercise improves psychosocial functioning and mental well-being [4,7,10,38,63-67]. 
In the context of MVAE-mediated cognitive benefits, 'acute exercise' which refers to a single bout of exercise, and 'chronic' or 'long-term' exercise that refers to multiple bouts of exercise spread over a longer period [16], trigger different neurobiological processes, with selective effects on sensory, short-term and long-term memory [16]; and different type of aerobic exercises do not all have the same effects on specific executive functions [17].

MVAE can mitigate central nervous system inflammatory processes by suppressing microglial responses to inflammatory stimuli [60,67]; and can moderate peripheral inflammatory responses by setting off reciprocal interactions between the neuroendocrine and the autonomic nervous systems [7,47]. MVAE also has the capacity to attenuate oxidative stresses which can both directly cause DNA damage and can dysregulate mechanisms of DNA repair $[62,68,69]$, and to suppress oxidative damage to central neural tissues, particularly to the hippocampus [70]. This is achieved by MVAE-mediated upregulation of activity of antioxidant enzymes, of enzymes degrading oxidatively damaged proteins, and of DNA repair enzymes. All these processes also promote clearance of cellular debris from the brain, thus promoting neuronal functionality and cognitive integrity [67].

Studies of Alzheimer disease models in transgenic mice show that aerobic exercise has the capability to downregulate the expression and function of genes encoding proinflammatory cytokines, thus attenuating the production of interleukin 1 beta (IL-1 $\beta$ ) and tumor necrosis factor alpha (TNF- $\alpha$ ) by hippocampal microglia. This appears to attenuate microglia-mediated neuroinflammatory and neurodegenerative processes $[19,70]$.

Physiologically, the $\mathrm{Wnt} / \beta$-catenin intracellular signaling pathway in the hippocampal tissue (of rats) takes part in the regulation of neuronal survival, plasticity, and in synaptic functionality, and as such, contributes to regulation of learning and memory. In the ageing brain, and with neurodegenerative processes, the $\mathrm{Wnt} / \beta$-catenin signaling pathway may be downregulated, contributing to a decline in cognitive performance, and to poor mental well-being; but aerobic exercise has the capacity to restore the functional integrity of $\mathrm{Wnt} / \beta$-catenin signaling, thereby reversing the cognitive impairment, and improving well-being [71].

Both animal and human studies show that MVAE induces central alterations in levels of noradrenalin, dopamine and serotonin, which can bring about states of arousal, pleasure and reward, and anxiety. It also mediates an increase in levels of endorphins and endocannabinoids which may promote euphoric, anxiolytic, sedative and analgesic effects [39]. Other central neurobiological agents induced directly or indirectly by aerobic exercise are neurotrophic mediators, such as brain-derived neurotrophic agents (BDNF), insulin-like growth factor (IGF-1), vascular endothelial growth factor (VEGF), neurotrophin-3, fibroblast growth factor-2 (FGF-2), nerve growth factor (NGF) and glial cellline derived neurotrophic factor (GDNF). All these biological agents play roles in the functional activity of the central nervous system, by facilitating neurogenesis, angiogenesis and synaptogenesis [39,72,73].

\section{The central endocannabinoid system in relation to aero- bic exercise, stress and mental well-being}

The endocannabinoid system comprises the endogenous lipid neurotransmitters $\mathrm{N}$-arachidonoyl ethanolamide (anandamide, AEA - this abbreviation anandamide derived from the Sanskrit word 'ananda' meaning bliss, joy, delight) and 2-arachidonoyl glycerol (2AG); the enzymes involved in their synthesis and degradation; and the cannabinoid receptors $\mathrm{CB} 1(\mathrm{CB} 1 \mathrm{R})$ and $\mathrm{CB} 2(\mathrm{CB} 2 \mathrm{R})$ [72,73]. $\mathrm{CB} 1 \mathrm{R}$ is broadly distributed throughout the central nervous system, most highly expressed in the hippocampus, amygdala, hypothalamus, cerebral cortex and in the spinal cord pain pathways; and CB2R is found mainly in peripheral sensory neurons and in activated microglia and astrocytes having the capacity to downregulate central neuroinflammatory processes [53,54,74-78].

Impulse activation of an endocannabinoid presynaptic neuron triggers the postsynaptic neuron to synthesize endocannabinoids which are released into the synaptic cleft, and then diffuse retrogradely to the presynaptic neuron, stimulating the cannabinoid receptors CB1R or CB2R situated in the presynaptic terminal, generating a neurobiological effect $[53,54]$. At most synapses, endocannabinoids inhibit the release of neurotransmitters from presynaptic terminals, thus modulating other neurotransmitter-induced excitatory or inhibitory postsynaptic responses $[53,74,79]$. Upon stimulation, AEA is rapidly biosynthesized 'on-demand' from membrane phospholipids, is not stored in vesicles [52], and is subsequently degraded by a specialized catabolic enzyme, the fatty acid amino hydrolase (FAAH) [53,57].

Maladaptive stress and anxiety responses are mediated, in part, by the neural circuitry within the basolateral amygdala (BLA). Under physiological conditions, AEA-CB1R signaling pathways in the BLA downregulates the BLA's neural excitability, probably owing to CB1R mediated presynaptic inhibition of glutamate release, with consequent diminution in glutaminergic excitatory transmission, thus providing regulatory tonic inhibition [78].

In response to stress, $\mathrm{CRH}$ via $\mathrm{CRH}$ receptor 1 (CRHR1) signaling upregulates FAAH activity within the BLA, bringing about a drop in AEA levels. Consequently, the tonic inhibitory AEA-CB1R signaling is dysregulated, facilitating excitatory transmission which promotes maladaptive stress and anxiety responses. Conversely, functional AEA-CB1R signaling in the amygdala, suppresses CRH-mediated neuroendocrine and behavioral maladaptive stress responses [57].

Unresolved chronic emotional stress and persistent anxiety mobilize and upregulate FAAH activity causing downregulation of AEA-CB1R signaling within the basolateral amygdala (BLA). This results not only in an increased BLA excitability, but also in activation of the HPA with increased levels of circulatory cortisol, further enhancing maladaptive stress and anxiety responses [78].

In certain brain regions, MVAE-induced CB1-mediated endocannabinoid signaling, together with other local neurotransmitters, regulates cognition, emotion, memory and mood [74,79]. For example, MVAE-mediated release of endocannabinoids promotes the activation of dopaminergic signaling circuits in the mesolimbic neural network, a dopaminergic pathway, triggering the operation of the brains' pleasure, reward and motivational pathways, promoting reward-driven behaviors, feelings of relaxation and sedation, and enhanced mood, all associated with positive mental well-being [39,42,72,80,81].

In addition, the endocannabinoid signaling system, and the brain derived neurotrophic factor (BDNF) signaling system, independently or jointly, may mediate some of the exercise-induced central neurobiological functions such as neurogenesis and synaptic plasticity, bringing about further MVAE-associated mental benefits $[43,54,77,82,83]$. Animal studies show that physical exercise increases the expression of AEA, CB1R and BDNF in the hippocampus with the activation of AEA-CB1R signaling, which promotes yet further BDNFmediated functional improvement of memory and cognition [77]. 
Furthermore, the endocannabinoid system has the capacity to mediate proliferation of neural progenitor cells, and their migration from their progenitor cell compartment in the hippocampus, and from other designated central neurological niches to their final destination in the brain [53]. Animal studies show that any type of movement upregulates neuronal activity within the hippocampus, and physical exercise, in particular, promotes the biosynthesis of local endocannabinoids and significantly enhances hippocampal AEA-CB1R signaling. Such exercise-induced upregulated AEA-CB1R activity, together with other exercise-induced neurotrophic factors, mediates proliferation of progenitor neural cells within the hippocampus [56].

Following their proliferation, differentiation and maturation, these progenitor neural cells play a significant role in repairing neuronal damage, and in supporting neurogenesis and synaptogenesis with the formation of new neural memory circuits in the hippocampus, leading to enhanced learning ability and memory capacity $[53,74,84]$. Other neuroprotective functions of the endocannabinoid system include downregulation of central immunoinflammatory reactions, excitotoxicity and oxidative and nitrosative processes; and modulation of mitochondrial dysfunctional activities which can cause neuronal damage $[74,75,84]$. Thus, overall, the endocannabinoid system plays an important role in the conservation of central neuronal integrity and longevity [85].

By stimulating peripheral cannabinoid receptors, exercise-induced endocannabinoids downregulate the activity of peripheral nociceptors and the release of inflammatory agents, thus generating an analgesic effect; and by stimulating central cannabinoid receptors at the level of the spinal cord and brainstem, endocannabinoids mediate central pain modulation $[39,42,73]$.

AEA-CB1R signaling, both tonically under basal conditions, and physically under stressful conditions inhibits HPA axis activities, thus contributing to the regulation of circulatory levels of glucocorticoids; but in the context of chronic psychosocial stress, persistent elevated levels of glucocorticoids downregulate CB1R signaling in the hypothalamus, leading to a compromised capacity of AEA-CB1R signaling to regulate HPX axis activities [52].

\section{Cross-stressor adaptation hypothesis}

The type of stress reactivity generated in response to psychosocial stressors, either adaptive or maladaptive, is determined by the functional activity of the autonomic nervous system and the neuroendocrine system, by how one appraises the relevant stressor, and by the effectiveness of one's psychological coping mechanisms $[9,27]$. Maladaptive responses to psychosocial stressors are characterized by exaggerated signs and symptoms of arousal (increased heart and breathing rates, salivary cortisol levels, etc.) and by increased emotional reactivity (irritability, impatience, intolerance, etc.), that negatively impact on mental well-being. As 'acute' vigorous aerobic exercises generate adaptive stress responses with signs and symptoms of physical arousal similar to those provoked by maladaptive psychosocial responses [8], it is likely that engaging in regular MVAE will familiarize one to the experience of (physical) arousal states. This may help (re) appraise psychosocial stressors as familiar routine challenges rather than threats, and consequently generate adaptive functional instead of maladaptive stress responses $[8,9,13]$. Thus, MVAE may mediate the development of 'cross-stressor adaptation', and as such, increase one's capacity to withstand stress in general, irrespective whether the stressors are physical or psychosocial in nature [8].
Through cognitive-behavioral therapy, subjects prone to exaggerated stress responses and negative affectivity, should be trained how to reappraise signs and symptoms of arousal as functional and adaptive, and not as threats. This may downregulate maladaptive stress responses and improve mental well-being $[8,9,11,13,14]$.

\section{Transient hyper frontality hypothesis}

During MVAE, most of the finite resources of mental energy are directed to brain regions which are essential for the exercise-related autonomic, motoric and sensory functioning. As a result, only limited resources of mental energy remain available for the activities of higher-cognitive functions in the prefrontal cortex which their operation are not essential during intense aerobic activity $[15,86,87]$. As some maladaptive upregulated stress-induced emotional responses (worry, rumination, anxiety, etc.) are driven, at least in part, by certain hyperactive neural circuits arising in the prefrontal cortex, transient downregulation of the activity of these circuits' secondary to exerciseinduced redistribution of mental energy in the brain, may curtail their hyperactivity. Consequently, some maladaptive stress-mediated emotional responses driven by the prefrontal cortex may be tapered, resulting in improved mental well-being $[15,86,88]$.

Furthermore, during endurance aerobic exercise, certain prefrontalrelated executive functions such as working memory, focused attention and self-control may be transiently weakened for the duration of the exercise thus compromising the ability to form complex judgments and make critical decisions [88]. Nevertheless, long-term, cognitive functions are improved following endurance physical exercise owing to the exercise-induced central neurostructural and neurochemical changes $[7,56,59,60,66,89]$.

\section{Epigenetics in relation to stress, aerobic exercise and mental well-being}

Epigenetic modifications refer to heritable changes in gene expression and function which are not owing to alterations in DNA sequences [22,90,91]. They act as transcriptional regulators, either enhancing or repressing gene expression, thus playing an important role in determining tissue phenotype and cellular functioning [20,90]. In general, properly balanced epigenetic modifications are essential for normal development and cellular differentiation and functioning, and in the context of neurobiology, for regulation of synaptic plasticity, neurotransmission, neurogenesis, for mental resilience and behavioral adaptation to psychological stressors, and for memory and learning. All of these contribute to mental well-being [18,92,93].

On the other hand, aberrant epigenetic modifications are associated with maladaptive stress responses, and with pathobiological processes implicated in the course of certain diseases, including the mental disturbances schizophrenia, bipolar disorder, Parkinson disease and Alzheimer disease $[18,94]$. Variations in epigenetic patterns can be induced by intrinsic (genetic), extrinsic (environmental) and stochastic factors [22,92]; and in general, DNA hypomethylation and histone hyperacetylation play essential roles in increased gene transcription [95].

The keystones of epigenetics are DNA methylation and histone modifications, which shape the molecular profile of one's dynamic epigenome. The epigenetic status is tissue-specific with some cell types being more resistant/receptive than others to environmentally induced epigenetic alterations, and the epigenetic status is also influenced by the nature of, and the level of exposure to the environmental agent $[92,96]$. 
Epigenetic modifications allow for adaptive changes in gene expression and function in response to environmental stimuli such as maternal care in infancy, psychosocial stress, infection, drugs, tobacco or alcohol habits, medications, nutrients and aerobic exercise, without any genomic alterations $[18,92,93]$. The different biological effects of the same environmental factors on different people may in part be owing to variations in their epigenetic patterns (polymorphism) $[22,94]$.

Psychosocial stressors may induce epigenetic modifications that downregulate the expression of genes mediating neurogenesis and synaptic plasticity, that upregulate genes promoting central inflammatory processes, and that impair the expression of genes mediating epigenetic regulation [22,97]. For example, different patterns of BDNF gene methylation, and BDNF genetic polymorphism are both relevant to the functional activity of BDNF protein, with specific genetic variants and specific methylation patterns having the potential, separately or together, to facilitate inflammatory processes and negatively to affect memory, learning and mental wellbeing, and to increase the risk of neurodegenerative diseases $[97,98]$. Hypermethylation of the promoter for the BDNF gene encoding the BDNF protein will downregulate or silence the BDNF gene expression and functional activity, particularly in the hippocampus where it is abundantly expressed [99]. The methylation profile of the BDNF gene is dynamic, and aberrant patterns can be corrected by medication, psychotherapy and indeed by regular MVAE [98].

Animal studies show that the expression of BDNF gene and its encoded protein in the hippocampus promotes dendritic arborization, and protects against stress-mediated hippocampal atrophy, and against stress-induced downregulation of neurogenesis and synaptogenesis; but some polymorphic variants of the BDNF gene are in fact associated with decreased production of its encoded protein, adversely affecting synaptic plasticity, diminishing neurogenesis and compromising cognitive functioning [58].

Perinatal maternal psychosocial stress-related adverse experiences may induce in the foetus or newborn aberrant epigenetic modifications, associated with dysregulation of expression of genes mediating stress responses, and malfunctioning of the neural circuits which mediate stress responses. These deviant stress-induced epigenetic modifications may remain embedded within the epigenome of the central nervous system, so that there will be some degree of lifelong impairment of stress responses, with decreased mental resilience and poor mental well-being $[22,93,100]$.

The glucocorticoid receptor (GR), widely distributed throughout the brain, plays a pivotal role in stress responses; and dysregulation of its activity is associated with maladaptive stress responses, and with certain mental disorders. In the brain, the biological outcome of the activation of GR, is region-and-neural circuit-specific, and it relies on the epigenetic pattern and on the variant of the GR gene [22]. Psychosocial stress-induced aberrant epigenetic modifications in the GR gene may lead to dysfunctional activity of the GR with consequent impairment of the negative feedback mechanism that regulates the HPA axis. This may result in hyperactivity of the HPA axis with persistent elevation of circulating cortisol levels and increased susceptibility to anxiety and depression, and to poor mental well-being [29,93].

For example, hypermethylation of the promoter of $\mathrm{Nr} 3 \mathrm{cl}$ GR gene in the hippocampus of adults who display maladaptive stress responses can be related to adverse emotional and abusive events that these adults had experienced in childhood [29,93]; and it appears that there is a link between maternal depression and having experienced disturbing stressful events during pregnancy on the one hand, and the pattern of hypermethylation at the $\mathrm{Nr} 3 \mathrm{cl}$ gene region, with associated increased levels of circulating cortisol observed in her newborn on the other hand [29]. In addition, there is evidence that epigenetic aberrations in relation to the $\mathrm{CRH}$ gene are also associated with susceptibility to stress, with post-stress maladaptive behavioral responses and with poor mental well-being $[58,93,100]$.

Increased levels of circulating glucocorticoids related to traumatically stressful early-life experiences are associated with subsequent demethylation of glucocorticoid receptor response elements (GREs). In turn, this demethylation of GREs promotes GR-mediated transcription of certain glucocorticoid responsive genes which regulate stress, immune responses and epigenetic modifications. In addition, stress-induced, glucocorticoid-mediated aberrant epigenetic alterations of the GR gene may increase the sensitivity of the receptor so that it upregulates transcription of glucocorticoid responsive genes without any substantial increase in the level of circulating cortisol [22].

In contrast to the detrimental epigenetic alterations induced by chronic psychosocial stress, moderate to vigorous intensity aerobic exercise (MVAE) induces epigenetic modifications that can give rise to an epigenetic configuration that supports the expression and functional activity of genes, such as the BDNF gene, that facilitate neurogenesis and synaptogenesis, thus providing neuroprotection and promoting cognitive functioning. Animal studies show that maternal aerobic exercise during pregnancy, induces in the offspring, epigenetic modifications that promote the expression of genes mediating synaptic plasticity and memory processing, culminating in enhanced cognitive performance [18]. Exercise affects DNA methylation in a gene-specific manner and the generated pattern of methlyation is determined by the degree of intensity of the exercise; and, in relation to cognitive functions, the same program of aerobic exercise does not equally influence one's various cognitive domains and may differently influence the same cognitive domain in different people [97,101].

It is not known to what extent one's genetic background, or the duration of regular MVAE, whether over years rather than over weeks or months, influences the nature of exercise-induced epigenetic alterations. It is also not known, once regular MVAE has in fact induced such epigenetic alterations, how much time elapses between these epigenetic alterations and the subsequent modified gene expression and the new protein profile. Yet another unknown is what tissuespecific mechanisms account for the variations in epigenetic patterns in different tissues in response to the same stimuli [101].

\section{Present global factors}

It would be remiss at this time not to refer to the Coronavirus Disease of 2019 (CoViD-19) pandemic at present afflicting the entire human world. Apart from the dreaded viral disease itself, the likely outcomes upon the individual of the associated lockdowns, the self- or governmentally imposed social distancing, the loss of personal social interaction, the curfews, the loss of work or severe curtailment of occupation, with consequent loss of remuneration, loneliness, and lack of inclination or opportunity to do any or sufficient physical exercise, have been dealt with at length above.

\section{Conclusion}

There is clear evidence of the positive effects of regular MVAE on mental well-being. Epigenetics (DNA methylation and histone modification)-induced reprogramming of genes is essential for the 
transduction of physical energy generated by aerobic exercise into cellular responses and neural signals that facilitate synaptic plasticity, neurogenesis and memory and learning, all of which play a critical role in promoting well balanced adaptive stress responses and mental wellbeing.

Exercise-induced neurochemical and neurostructural refinements to the functional activities of the HPA, sympatho-neural and sympathoadrenomedullary axes may enhance sleep quality, boost resources of mental energy, improve cognitive functions and mood, and moderate negative emotional perceptions, all of which improve mental wellbeing. The reasons why the benefits of MVAE are greater for some cognitive domains relative to others, which age groups benefit most from regular MVAE, and whether or not regular engagement with MVAE in adolescence and early adulthood provide neuroprotection from future cognitive decline in adulthood and old age, are questions that still need to be answered.

\section{References}

1. Piercy KL, Troiano RP (2018) Physical Activity Guidelines for Americans From the US Department of Health and Human Services. Circ Cardiovasc Qual Outcomes 11: e005263. [Crossref]

2. Patel H, Alkhawam H, Madanieh R, Shah N, Kosmas CE, et al. (2017) Aerobic vs anaerobic exercise training effects on the cardiovascular system. World J Cardiol 9: 134-138. [Crossref]

3. Chamari K, Padulo J (2015) 'Aerobic' and 'Anaerobic' terms used in exercise physiology: a critical terminology reflection. Sports Med Open 1: 9. [Crossref]

4. Mandolesi L, Polverino A, Montuori S, Foti F, Ferraioli G, et al. (2018) Effects of Physical Exercise on Cognitive Functioning and Wellbeing: Biological and Psychological Benefits. Front Psychol 9: 509. [Crossref]

5. Team AHAE (2018) American Heart Association Recommendations for Physical Activity in Adults and Kids. [Available at: https://www.heart.org/en/healthy-living/ fitness/fitness-basics/aha-recs-for-physical-activity-in-adults].

6. Norris R, Carroll D, Cochrane R (1992) The effects of physical activity and exercise training on psychological stress and well-being in an adolescent population. $J$ Psychosom Res 36: 55-65. [Crossref]

7. Silverman MN, Deuster PA (2014) Biological mechanisms underlying the role of physical fitness in health and resilience. Interface Focus 4: 20140040. [Crossref]

8. Childs E, de Wit H (2014) Regular exercise is associated with emotional resilience to acute stress in healthy adults. Front Physiol 5: 161. [Crossref]

9. Jacquart J, Papini S, Freeman Z, Bartholomew JB, Smitsa JAJ (2020) Using exercise to facilitate arousal reappraisal and reduce stress reactivity: A randomized controlled trial. Mental Health Phys Activity 18: 100324.

10. Anderson E, Shivakumar G (2013) Effects of exercise and physical activity on anxiety. Front Psychiatry 4: 27. [Crossref]

11. Arvidson E, Dahlman AS, Börjesson M, Gullstrand L, Jonsdottir IH (2018) Exercise training and physiological responses to acute stress: study protocol and methodological considerations of a randomised controlled trial. BMJ Open Sport Exerc Med 4: e000393. [Crossref]

12. Tamminen N, Reinikainen J, Appelqvist-Schmidlechner K, Borodulin K, MäkiOpas T, et al. (2020) Associations of physical activity with positive mental health: A population-based study. Mental Health Phys Activity 18: 100319.

13. Herbert C, Meixner F, Wiebking C, Gilg V (2020) Regular Physical Activity, ShortTerm Exercise, Mental Health, and Well-Being Among University Students: The Results of an Online and a Laboratory Study. Front Psychol 11: 509. [Crossref]

14. Gerber M, Pühse U (2009) Review article: do exercise and fitness protect against stressinduced health complaints? A review of the literature. Scand J Public Health 37: 801819. [Crossref]

15. Dietrich A (2003) Functional neuroanatomy of altered states of consciousness: the transient hypofrontality hypothesis. Conscious Cogn 12: 231-256. [Crossref]

16. Roig M, Nordbrandt S, Geertsen SS, Nielsen JB (2013) The effects of cardiovascular exercise on human memory: a review with meta-analysis. Neurosci Biobehav Rev 37: 1645-1666. [Crossref]
17. Wang CH, Tsai CL (2016) Physical Activity Is Associated With Greater Visuospatial Cognitive Functioning Regardless of the Level of Cognitive Load in Elderly Adults. $J$ Sport Exerc Psychol 38: 69-81. [Crossref]

18. Fernandes J, Arida RM, Gomez-Pinilla F (2017) Physical exercise as an epigenetic modulator of brain plasticity and cognition. Neurosci Biobehav Rev 80: 443-456. [Crossref]

19. Daniele S, Giacomelli C, Loprinzi PD, Franzoni F (2020) Editorial: Physical Activity: Epigenetic and Metabolic Regulation of Brain Aging. Front Aging Neurosci 12: 195. [Crossref]

20. Sailani MR, Halling JF, Møller HD, Lee H, Plomgaard P, et al. (2019) Lifelong physical activity is associated with promoter hypomethylation of genes involved in metabolism, myogenesis, contractile properties and oxidative stress resistance in aged human skeletal muscle. Sci Rep 9: 3272. [Crossref]

21. Bertogliat MJ, Morris-Blanco KC, Vemuganti R (2020) Epigenetic mechanisms of neurodegenerative diseases and acute brain injury. Neurochem Int 133: 104642. [Crossref]

22. Klengel T, Binder EB (2015) Epigenetics of Stress-Related Psychiatric Disorders and Gene $\times$ Environment Interactions. Neuron 86: 1343-1357. [Crossref]

23. Bell SL, Audrey S, Gunnell D, Cooper A, Campbell R (2019) The relationship between physical activity, mental wellbeing and symptoms of mental health disorder in adolescents: a cohort study. Int J Behav Nutr Phys Act 16: 138. [Crossref]

24. Diener E, Ryan K (2009) Subjective well-being: A general overview. South African J Psychol 39: 391-406.

25. Doré I, Sylvester B, Sabiston C, Sylvestre M, O’Loughlin J, et al. (2020) Mechanisms underpinning the association between physical activity and mental health in adolescence: a 6-year study. Int J Behavioral Nutrition Phy Activity 17: 9.

26. Van Tongeren DR, Davis DE, Hooka JN, Witvliet C (2019) Humility. Curr Dir Psychol Sci 28: 463-468.

27. Krause N, Pargament KI, Hill PC, Ironson G (2016) Humility, stressful life events, and psychological well-being: Findings from the landmark spirituality and health survey. $J$ Posit Psychol 11: 499-510.

28. Herman JP (2013) Neural control of chronic stress adaptation. Front Behav Neurosci 7: 61. [Crossref]

29. Dadds MR, Moul C, Hawes DJ, Mendoza Diaz A, Brennan J (2015) Individual Differences in Childhood Behavior Disorders Associated With Epigenetic Modulation of the Cortisol Receptor Gene. Child Dev 86: 1311-1320. [Crossref]

30. Martela F, Pessi AB (2018) Significant Work Is About Self-Realization and Broader Purpose: Defining the Key Dimensions of Meaningful Work. Front Psychol 9: 363. [Crossref]

31. Martela F, Riekki TJJ (2018) Autonomy, Competence, Relatedness, and Beneficence: A Multicultural Comparison of the Four Pathways to Meaningful Work. Front Psychol 9: 1157. [Crossref]

32. Hartzband P, Groopman J (2020) Physician Burnout, Interrupted. N Engl J Med 382: 2485-2487. [Crossref]

33. Gagné M, Deci EL (2005) Self-determination theory and work motivation. $J$ Organizational Behav 26: 331-362.

34. Legault L (2016) The need for autonomy. Encyclopedia of Personality and Individual Differences, Springer, New York, NY, pp: 1120-1122.

35. Legault L (2017) The need for competence. Encyclopedia of Personality and Individual Differences, Springer, New York, NY, pp: 973-978.

36. Keller H. Psychological autonomy and hierarchical relatedness as organizers of developmental pathways. Philos Trans R Soc Lond B Biol Sci 371: 20150070. [Crossref]

37. Weinstein N, Ryan RM (2010) When helping helps: autonomous motivation for prosocial behavior and its influence on well-being for the helper and recipient. J Pers Soc Psychol 98: 222-244. [Crossref]

38. Alexandratos K, Barnett F, Thomas Y (2012) The impact of exercise on the mental health and quality of life of people with severe mental illness: a critical review. $\mathrm{Br} \mathrm{J}$ Occup Ther 75: 48-60.

39. Rahman M, Lehmann-Waldau F, Werfalli R (2018) Current Evidence and Use of Physical Activity in the Treatment of Mental Illness: A Literature Review. Aust Nz J Psychiat 52: 83-83.

40. Caldwell Hooper AE, Bryan AD, Hagger MS (2014) What keeps a body moving? The brain-derived neurotrophic factor val66met polymorphism and intrinsic motivation to exercise in humans. J Behav Med 37: 1180-1192. [Crossref] 
41. Dietrich A, Stoll O (2010) Effortless attention, hypofrontality, and perfectionism. Effortless attention: A new perspective in the cognitive science of attention and action. pp: $159-178$.

42. Dietrich A, McDaniel WF (2004) Endocannabinoids and exercise. Br J Sports Med 38: 536-41. [Crossref]

43. Heyman E, Gamelin FX, Goekint M, Piscitelli F, Roelands B, et al. (2012) Intense exercise increases circulating endocannabinoid and BDNF levels in humans--possible implications for reward and depression. Psychoneuroendocrinology 37: 844-851. [Crossref]

44. Mastorakos G, Pavlatou M, Diamanti-Kandarakis E, Chrousos GP (2005) Exercise and the stress system. Hormones (Athens) 4: 73-89. [Crossref]

45. Ulrich-Lai YM, Herman JP (2009) Neural regulation of endocrine and autonomic stress responses. Nat Rev Neurosci 10: 397-409. [Crossref]

46. Carter JR, Goldstein DS (2015) Sympathoneural and adrenomedullary responses to mental stress. Compr Physiol 5: 119-146. [Crossref]

47. Feller L, Khammissa RAG, Ballyram R, Chandran R (2019) Chronic Psychosocial Stress in Relation to Cancer. Middle East J Cancer 10: 1-8.

48. Feller L, Feller G, Ballyram T, Chandran R, Lemmer J, et al. (2020) Interrelations between pain, stress and executive functioning. Br J Pain 14: 188-194. [Crossref]

49. Becker L, Rohleder N (2019) Time course of the physiological stress response to an acute stressor and its associations with the primacy and recency effect of the serial position curve. PLoS One 14: e213883. [Crossref]

50. Farias-Godoy A, Chan S, Claydon VE, Ignaszewski A, Mendell J, et al. (2018) The Impact of Reduced Cardiac Rehabilitation on Maximal Treadmill Exercise Time: A RANDOMIZED CONTROLLED TRIAL. J Cardiopulm Rehabil Prev 38: 24-30. [Crossref]

51. Goldstein DS (2010) Adrenal responses to stress. Cell Mol Neurobiol 30: 1433-1440. [Crossref]

52. Crosby KM, Bains JS (2012) The intricate link between glucocorticoids and endocannabinoids at stress-relevant synapses in the hypothalamus. Neuroscience 204: 31-37. [Crossref]

53. Kalant H (2014) Effects of cannabis and cannabinoids in the human nervous system. The effects of drug abuse on the human nervous system. Elsevier, pp.387-422.

54. Heijnen S, Hommel B, Kibele A, Colzato LS (2016) Neuromodulation of Aerobic Exercise-A Review. Front Psychol 6: 1890. [Crossref]

55. Khammissa R, Ballyram R, Wood N, Lemmer J, Feller L (2016) Glucocorticosteroids in the treatment of immune mediated oral diseases. South African Dental J 71: 62-67.

56. Hill MN, McLaughlin RJ, Bingham B, Shrestha L, Lee TT, et al. (2010) Endogenous cannabinoid signaling is essential for stress adaptation. Proc Natl Acad Sci U S A 107: 9406-9411. [Crossref]

57. Gray JM, Vecchiarelli HA, Morena M, Lee TT, Hermanson DJ, et al. (2015) Corticotropin-releasing hormone drives anandamide hydrolysis in the amygdala to promote anxiety. J Neurosci 35: 3879-3892. [Crossref]

58. Yuen EY, Wei J, Yan Z (2017) Molecular and Epigenetic Mechanisms for the Complex Effects of Stress on Synaptic Physiology and Cognitive Functions. Int $J$ Neuropsychopharmacol 20: 948-955. [Crossref]

59. Hong JS, Kim SM, Kang KD, Han DH, Kim JS, et al. (2020) Effect of physical exercise intervention on mood and frontal alpha asymmetry in internet gaming disorder. Mental Health Phys Activity 18: 100318

60. Beckman D, Santos LE (2013) The importance of serotonin in exercise-induced adult neurogenesis: new evidence from Tph2-/- mice. J Neurosci 33: 14283-14284. [Crossref]

61. Erickson KI, Hillman C, Stillman CM, Ballard RM, Bloodgood B, et al. (2019) Physical Activity, Cognition, and Brain Outcomes: A Review of the 2018 Physical Activity Guidelines. Med Sci Sports Exerc 51: 1242-1251. [Crossref]

62. Matta Mello Portugal E, Cevada T, Sobral Monteiro-Junior R, Teixeira Guimarães T, da Cruz Rubini E, et al. (2013) Neuroscience of exercise: from neurobiology mechanisms to mental health. Neuropsychobiology 68: 1-14. [Crossref]

63. Sharma A, Madaan V, Petty FD (2006) Exercise for mental health. Prim Care Companion J Clin Psychiatry 8: 106. [Crossref]

64. Meyer JD, Crombie KM, Cook DB, Hillard CJ, Koltyn KF (2019) Serum Endocannabinoid and Mood Changes after Exercise in Major Depressive Disorder. Med Sci Sports Exerc 51: 1909-1917. [Crossref]
65. Zhang B, Liu Y, Zhao M, Meng X, Deng Y, et al. (2020) Differential effects of acute physical activity on executive function in preschoolers with high and low habitual physical activity levels. Mental Health Phys Activity 18: 100326.

66. Erickson KI, Voss MW, Prakash RS, Basak C, Szabo A, et al. (2011) Exercise training increases size of hippocampus and improves memory. Proc Natl Acad Sci U S A 108 3017-3022. [Crossref]

67. Daniele S, Giacomelli C, Martini C (2018) Brain ageing and neurodegenerative disease: The role of cellular waste management. Biochem Pharmacol 158: 207-216. [Crossref]

68. de Sousa CV, Sales MM, Rosa TS, Lewis JE, de Andrade RV, et al. (2017) The Antioxidant Effect of Exercise: A Systematic Review and Meta-Analysis. Sports Med 47: 277-293. [Crossref]

69. Lin TW, Kuo YM (2013) Exercise benefits brain function: the monoamine connection. Brain Sci 3: 39-53. [Crossref]

70. Zhang X, He Q, Huang T, Zhao N, Liang F, Xu B, et al. (2019) Treadmill Exercise Decreases A $\beta$ Deposition and Counteracts Cognitive Decline in APP/PS1 Mice, Possibly via Hippocampal Microglia Modifications. Front Aging Neurosci 11: 78. [Crossref]

71. Chen D, Zhang Y, Zhang M, Chang J, Zeng Z, et al. (2020) Exercise Attenuates Brain Aging by Rescuing Down-Regulated Wnt/ $\beta$-Catenin Signaling in Aged Rats. Front Aging Neurosci 12: 105. [Crossref]

72. Stone NL, Millar SA, Herrod PJJ, Barrett DA, Ortori CA, et al. (2018) An Analysis of Endocannabinoid Concentrations and Mood Following Singing and Exercise in Healthy Volunteers. Front Behav Neurosci 12: 269. [Crossref]

73. Tantimonaco M, Ceci R, Sabatini S, Catani MV, Rossi A, et al. (2014) Physical activity and the endocannabinoid system: an overview. Cell Mol Life Sci 71: 2681-2698. [Crossref]

74. Cassano T, Calcagnini S, Pace L, De Marco F, Romano A, et al. (2017) Cannabinoid Receptor 2 Signaling in Neurodegenerative Disorders: From Pathogenesis to a Promising Therapeutic Target. Front Neurosci 11: 30. [Crossref]

75. Javed H, Azimullah S, Haque ME, Ojha SK (2016) Cannabinoid Type 2 (CB2) Receptors Activation Protects against Oxidative Stress and Neuroinflammation Associated Dopaminergic Neurodegeneration in Rotenone Model of Parkinson's Disease. Front Neurosci 10: 321. [Crossref]

76. Haider A, Müller Herde A, Slavik R, Weber M, Mugnaini C, et al. (2016) Synthesis and Biological Evaluation of Thiophene-Based Cannabinoid Receptor Type 2 Radiotracers for PET Imaging. Front Neurosci 10: 350. [Crossref]

77. Ferreira-Vieira TH, Bastos CP, Pereira GS, Moreira FA, Massensini AR (2014) A role for the endocannabinoid system in exercise-induced spatial memory enhancement in mice. Hippocampus 24: 79-88. [Crossref]

78. Gunduz-Cinar O, Hill MN, McEwen BS, Holmes A (2013) Amygdala FAAH and anandamide: mediating protection and recovery from stress. Trends Pharmacol Sci 34: 637-644. [Crossref]

79. Contino M, Capparelli E, Colabufo NA, Bush AI (2017) Editorial: The CB2 Cannabinoid System: A New Strategy in Neurodegenerative Disorder and Neuroinflammation. Front Neurosci 11: 196. [Crossref]

80. Esch T, Stefano GB (2004) The neurobiology of pleasure, reward processes, addiction and their health implications. Neuro Endocrinol Lett 25: 235-251. [Crossref]

81. Cordeiro LMS, Rabelo PCR, Moraes MM, Teixeira-Coelho F, Coimbra CC, et al (2017) Physical exercise-induced fatigue: the role of serotonergic and dopaminergic systems. Braz J Med Biol Res 50: e6432. [Crossref]

82. Yeh ML, Selvam R, Levine ES (2017) BDNF-induced endocannabinoid release modulates neocortical glutamatergic neurotransmission. Synapse 71: 10.1002/ syn.21962. [Crossref]

83. Gangarossa G, Perez S, Dembitskaya Y, Prokin I, Berry H, Venance L (2020) BDNF Controls Bidirectional Endocannabinoid Plasticity at Corticostriatal Synapses. Cereb Cortex 30: 197-214. [Crossref]

84. Aso E, Ferrer I (2016) CB2 Cannabinoid Receptor As Potential Target against Alzheimer's Disease. Front Neurosci 10: 243. [Crossref]

85. Navarro G, Morales P, Rodríguez-Cueto C, Fernández-Ruiz J, Jagerovic N, et al. (2016) Targeting Cannabinoid CB2 Receptors in the Central Nervous System. Medicinal Chemistry Approaches with Focus on Neurodegenerative Disorders. Front Neurosci 10: 406. [Crossref]

86. Dietrich A (2006) Transient hypofrontality as a mechanism for the psychological effects of exercise. Psychiatry Res 145: 79-83. [Crossref] 
Feller L (2021) The interrelation between aerobic exercise, mental well-being, stress response and epigenetics

87. Raichlen DA, Foster AD, Gerdeman GL, Seillier A, Giuffrida A (2012) Wired to run: exercise-induced endocannabinoid signaling in humans and cursorial mammals with implications for the 'runner's high'. J Exp Biol 215: 1331-1336. [Crossref]

88. Dietrich A, Sparling PB (2004) Endurance exercise selectively impairs prefrontaldependent cognition. Brain Cogn 55: 516-524. [Crossref]

89. Erickson KI, Hillman C, Stillman CM, Ballard RM, Bloodgood B, et al. (2019) Physical Activity, Cognition, and Brain Outcomes: A Review of the 2018 Physical Activity Guidelines. Med Sci Sports Exerc 51: 1242-1251. [Crossref]

90. Denham J, Marques FZ, O'Brien BJ, Charchar FJ (2014) Exercise: putting action into our epigenome. Sports Med 44: 189-209. [Crossref]

91. Feller L, Bouckaert M, Chikte UM, Wood NH, Khammissa RA, et al. (2010) A short account of cancer--specifically in relation to squamous cell carcinoma. SADJ 65: 322324. [Crossref]

92. Aguilera O, Fernández AF, Muñoz A, Fraga MF (2010) Epigenetics and environment: a complex relationship. J Appl Physiol (1985) 109: 243-251. [Crossref]

93. Stankiewicz AM, Swiergiel AH, Lisowski P (2013) Epigenetics of stress adaptations in the brain. Brain Res Bull 98: 76-92. [Crossref]

94. Peedicayil J (2007) The role of epigenetics in mental disorders. Indian J Med Res 126: 105-111. [Crossref]
95. McGee SL, Hargreaves M (2019) Epigenetics and Exercise. Trends Endocrinol Metab 30: 636-645. [Crossref]

96. Radtke KM, Schauer M, Gunter HM, Ruf-Leuschner M, Sill J, et al. (2015) Epigenetic modifications of the glucocorticoid receptor gene are associated with the vulnerability to psychopathology in childhood maltreatment. Transl Psychiatry 5: e571. [Crossref]

97. Wagner MA, Erickson KI, Bender CM, Conley YP (2020) The Influence of Physica Activity and Epigenomics On Cognitive Function and Brain Health in Breast Cancer. Front Aging Neurosci 12: 123. [Crossref]

98. Voisey J, Lawford B, Bruenig D, Harvey W, Morris CP, et al. (2019) PTSD Initiative. Differential BDNF methylation in combat exposed veterans and the association with exercise. Gene 698: 107-112. [Crossref]

99. Ntanasis-Stathopoulos J, Tzanninis JG, Philippou A, Koutsilieris M (2013) Epigenetic regulation on gene expression induced by physical exercise. J Musculoskelet Neuronal Interact 13: 133-146. [Crossref]

100. McEwen BS (2016) In pursuit of resilience: stress, epigenetics, and brain plasticity Ann N Y Acad Sci 1373: 56-64. [Crossref]

101. Voisin S, Eynon N, Yan X, Bishop DJ (2015) Exercise training and DNA methylation in humans. Acta Physiol (Oxf) 213: 39-59. [Crossref] 